\title{
CDS wide slit time-series of EUV coronal bright points
}

\author{
I. Ugarte-Urra ${ }^{1}$, J. G. Doyle ${ }^{1}$, V. M. Nakariakov ${ }^{2}$, and C. R. Foley ${ }^{3}$ \\ 1 Armagh Observatory, College Hill, Armagh BT61 9DG, N. Ireland \\ e-mail: iuu@star.arm.ac.uk \\ 2 Physics Department, University of Warwick, Coventry, CV4 7AL, UK \\ 3 Mullard Space Science Laboratory, University College London, Holmbury St. Mary, Dorking, Surrey RH5 6NT, UK
}

Received 9 April 2004 / Accepted 10 June 2004

\begin{abstract}
Wide slit $\left(90^{\prime \prime} \times 240^{\prime \prime}\right)$ movies of four Extreme Ultraviolet coronal bright points (BPs) obtained with the Coronal Diagnostic Spectrometer (CDS) on board the Solar and Heliospheric Observatory (SoHO) have been inspected. The wavelet analysis of the He I $584.34 \AA$, O v $629.73 \AA$ and Mg viI/Ix $368 \AA$ time-series confirms the oscillating nature of the BPs, with periods ranging between 600 and $1100 \mathrm{~s}$. In one case we detect periods as short as $236 \mathrm{~s}$. We suggest that these oscillations are the same as those seen in the chromospheric network and that a fraction of the network bright points are most likely the cool footpoints of the loops comprising coronal bright points. These oscillations are interpreted in terms of global acoustic modes of the closed magnetic structures associated with BPs.
\end{abstract}

Key words. Sun: oscillations - Sun: corona - Sun: transition region - Sun: chromosphere - Sun: UV radiation Sun: magnetic fields

\section{Introduction}

Coronal oscillations have been studied for more than thirty years in several wavelength ranges (Aschwanden 2003, and references therein). In recent years, observations made with the Transition Region And Coronal Explorer (TRACE) and instruments onboard the Solar and Heliospheric Observatory (SoHO) have provided evidence of these oscillations in different scenarios: sunspots, TRACE loops, polar plumes, etc. (e.g., O'Shea et al. 2001; Banerjee et al. 2002; Marsh et al. 2002; Deforest \& Gurman 1998). Another good candidate to host coronal oscillations are Extreme Ultraviolet (EUV) coronal bright points (BPs). They are identified as small (10-30 Mm) regions of enhanced emission over the quiet Sun and coronal holes. They comprise tiny loops (Sheeley \& Golub 1979) and lie over the network boundaries of the supergranular cells (Egamberdiev 1983), which are characterized for their oscillating nature (Dame et al. 1984). BPs are the result of the interaction of opposite magnetic polarities with magnetic reconnection likely to play a major role in the BP appearance (Priest et al. 1994). In this respect, BPs have been seen to disappear at coronal temperatures after the full cancellation of one of the magnetic polarities (Madjarska et al. 2003). These authors also commented on small-scale brightenings within the BP which showed velocity variations in the range $3-6 \mathrm{~km} \mathrm{~s}^{-1}$. The analysis of the transition region line S vi $933 \AA$ flux fluctuations showed clear evidence for a period just under $500 \mathrm{~s}$ (Ugarte-Urra et al. 2004). These authors commented on oscillations seen in another BP in O v $629 \AA$ which looked like a damped wave.
Over the last years of successful space observations it has become clear that it is necessary to combine observations of the same phenomenon from different instruments with different capabilities. In the case of BPs, the role of the magnetic field is crucial in the evolution of the features and whenever possible its study should complement the imaging and spectral analysis. Here, we extend the BP oscillation study by looking at timeseries data taken with the Coronal Diagnostic Spectrometer in its wide-slit mode, coupled with data from other instruments on board SoHO. Observations are discussed in Sects. 2 and 3 describes the wavelet analysis results of the study of four BPs, while Sect. 4 establishes a comparison with the magnetic field evolution in one of the cases. In Sect. 5, we discuss the results in the context of the chromospheric oscillations in the network, as well as, the possible wave mechanisms and reconnection models. The conclusions are given in Sect. 6 .

\section{Observations}

\section{1. $C D S$}

The Coronal Diagnostic Spectrometer (CDS) onboard SoHO was designed to determine the characteristics of the solar atmosphere plasma through the study of the emission lines characteristics in the 150-800 Å EUV spectral range (Harrison et al. 1995). Two spectrometers, a normal (NIS) and a grazing incidence (GIS), can be used for that purpose. In the case of NIS, the selection of a wide slit $\left(90^{\prime \prime} \times 240^{\prime \prime}\right)$ allows one to obtain high temporal cadence images of a target at the expense of spectral resolution. Therefore, we can create a time-series 
Table 1. Details of the CDS wide slit $\left(90^{\prime \prime} \times 240^{\prime \prime}\right)$ observational programs, including their identification number, acronym, date and time of observation, center coordinates of the slit for that time in arcseconds, total duration in minutes, exposure time in seconds for each of the images and average time cadence in seconds with its standard deviation.

\begin{tabular}{ccccccc}
\hline \hline Program & Acronym & Date - Starting time & Coordinates ["] & Duration [min] & Exp. time [s] & Avg. cadence [s] \\
\hline s6744 & CHJET_v1 & $1997 / 01 / 20-13: 23$ UT & $0,-992$ & 128 & 60 & $70.4 \pm 1.6$ \\
s6829 & CHJET_v1 & $1997 / 01 / 30-09: 30$ UT & $-31,-982$ & 128 & 60 & $70.4 \pm 1.6$ \\
s6840 & CHJET_v1 & $1997 / 01 / 31-08: 02$ UT & $-31,-984$ & 93 & 60 & $70.0 \pm 1.7$ \\
s6697 & MOVIE_V1/v3 & $1997 / 01 / 16-08: 02$ UT & 0,0 & 218 & 25 & $31.2 \pm 0.4$ \\
\hline
\end{tabular}

of a specific solar region at a cadence of 1 image every $30 \mathrm{~s}$, as seen by different spectral lines with different formation temperatures. As images produced by close lines would overlap, we are constrained to the study of isolated and unblended lines. This mode has proven to be very useful in the study of different solar features: blinkers, coronal jets, prominences, quiet Sun network and sunspots. The present BP observations were done in 1997 (see Table 1), using the wide slit in a sit-and-stare mode, i.e. the pointing is kept fixed and the plasma moves under the slit. Three bright lines of the NIS spectral range were used: He I $584.34 \AA$, O v $629.73 \AA$ and Mg ix $368.07 \AA$. Their formation temperature in ionization equilibrium is respectively, in logarithmic scale, 4.5, 5.4 and 6.0 (Mazzotta et al. 1998; Young et al. 2003). The Mg Ix line is blended with a Mg VII 367.67 A line which has a formation temperature of 5.9, therefore the image of the slit centered at $368 \AA$ is the product of the BP emission in these two temperatures. Table 1 shows the details of these observations. Each NIS pixel corresponds to $1.68^{\prime \prime}$ square, although the spatial resolution of CDS is closer to $6 "$ (Pauluhn et al. 1999). A standard reduction was applied to the images in order to correct for bias, flat-field, cosmic rays and instrumental effects.

The BPs were visually identified in the Mg VII/IX window as compact regions with an enhanced emission over the surrounding corona. However, we note that network elements and blinkers can both have a coronal response (Gallagher et al. 1998). Leaving aside, for the moment, the possible link that could exist between both phenomena, we confirmed the BP nature by inspecting images from the Extreme ultraviolet Imaging Telescope (EIT). In these images, BPs are seen as point-like or loop-like structures that last for several hours (Zhang et al. 2001). All of them last for the whole CDS sequence, which varied between 93 and $218 \mathrm{~min}$.

\subsection{EIT}

Images from the Extreme ultraviolet Imaging Telescope (EIT) were used to identify and follow the life evolution of the BPs. The BP corresponding to the CDS dataset s6744 can be identified at $\approx 13: 00$ UT on 20 January 1997 in the 171, 195 and $284 \AA$ bandpasses, and remains still visible at 15:09 in the $195 \AA$ band. For the s6829 dataset, the BP was a fuzzy enhanced region at 7:00 UT in the $195 \AA$ band that slowly became brighter and more recognizable by 9:00 UT. Its intensity was still increasing at 13:04 UT. For the s6840 dataset, the BP appeared some time between 5:00 UT and 7:00 UT (visible in
171, 195 and $284 \AA$ bands). By 9:00 UT it had reached its maximum brightness in 195 ̊ and was still visible at 13:00 UT, although fainter. The BP in dataset s6697 was visible for several hours (at least from 8 UT to 14 UT).

\section{3. $M D I$}

MDI (Michelson Doppler Imager) high resolution magnetograms $\left(0.6^{\prime \prime} / \mathrm{pixel}\right)$ were also analyzed for the BP observed on January 161997 . They cover the whole CDS observational period with a cadence of one exposure per minute. The magnetograms were corrected for differential solar rotation and for the geometrical projection of the line-of-sight magnetic flux (Chae et al. 2001; Hagenaar 2001). In order to increase the signal to noise, every five consecutive exposures were averaged resulting in a final cadence of $\sim 5 \mathrm{~min}$.

\section{Wavelet analysis}

BPs are characterized by a high flux variability in EUV emission (Habbal \& Withbroe 1981; Habbal et al. 1990). Our objective in this paper is to extend to more cases the periodicity analysis done by Ugarte-Urra et al. (2004) for two BPs and try to determine whether oscillations are a common feature in their brightness evolution and whether they show a coronal response. We are aware that oscillations in the quiet Sun network have been found to be intermittent (Banerjee et al. 2001), therefore, we are interested in the use of a technique that allows the study of non-stationary signals along the time-series. The wavelet analysis is a technique that permits the extraction of the relevant signals present in a time-series by convolving the series with a wavelet function. The result is a two dimensional (time and period) power spectrum, like that shown in the center panel of Fig. 1, where the regions with higher power values, and therefore higher response to the wavelet function, are plotted in darker gray. The analysis suffers from edge effects due to the fact that we are dealing with time-series of a finite length. The region in the power spectrum under this influence is called the cone of influence (COI), which is indicated by the crosshatched regions. Periodicities found inside the cor are unreliable and will not be considered. We have used the software and definitions provided by Torrence \& Compo (1998) and the wavelet function chosen for the convolution is the Morlet, which provides the best compromise between time and period resolution (De Moortel \& Hood 2000). An important part of the analysis is to determine the relevance of the peaks in the wavelet power, associated with specific periods and specific 

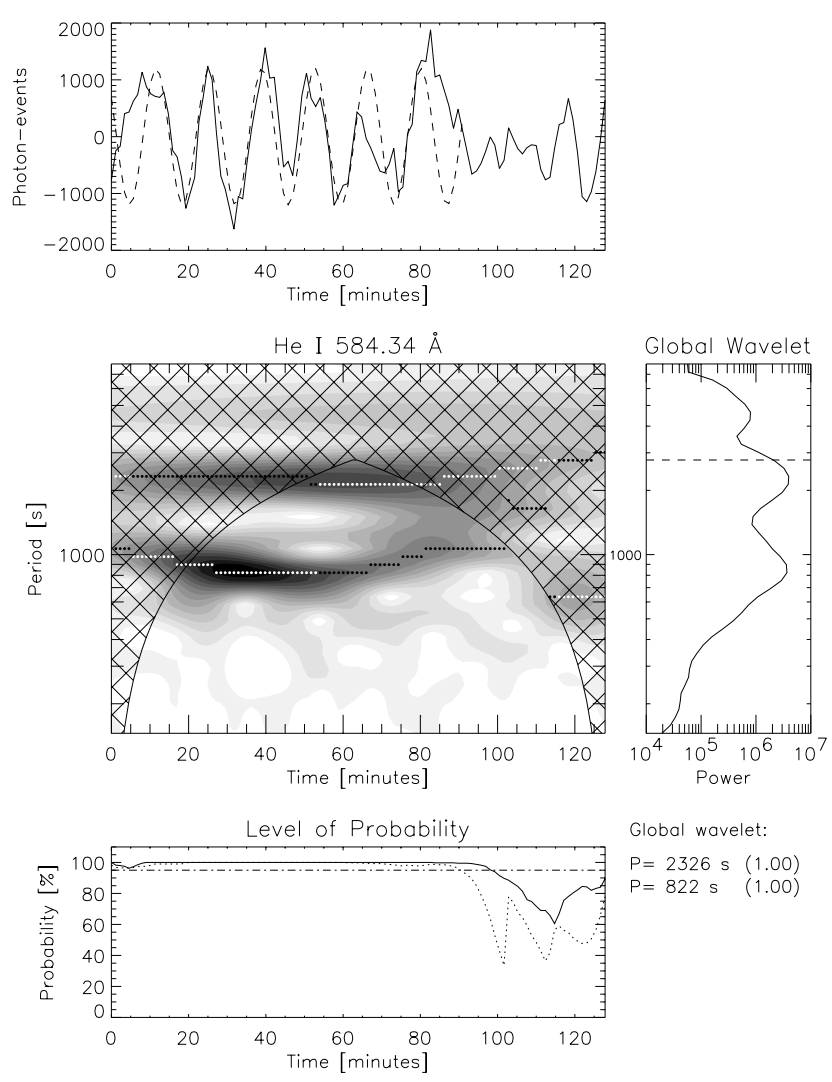

Global wavelet:

$P=2326 s(1.00)$ $\mathrm{P}=822 \mathrm{~s} \quad(1.00)$

Fig. 1. Wavelet analysis results for the He I $584 \AA$ time series of the BP observed on January 20, 1997. Top panel: time variation of the number of counts after detrending; a sine function with a period of $822 \mathrm{~s}$, corresponding to the peak in the wavelet analysis, is overplotted. Middle panels: wavelet power spectrum with the cone of influence over-plotted (cross-hatched region) and global wavelet. The dashed line represents the maximum period outside the cone of influence. The periods corresponding to the first two maximums in the global wavelet are indicated below the graph, together with the associated level of probability in brackets. Bottom panel: variation in time of the probability associated with the two strongest peaks in each time slice. The solid line is associated to the first maximum location (white dots on the power spectrum) and the dotted line to the second maximum (black dots). The $95 \%$ confidence level is given by the dotdashed line.

moments of time. A randomization method is used to estimate the significance levels (O'Shea et al. 2001). The basic assumption of the method is that if there is no periodicity in the timeseries, any order of the intensity values would be as likely as any other. By comparing the power spectrum obtained from the observed series to the one obtained from each of the permutations in time of the intensity values, we can estimate the probability $p$ that a certain peak in the power spectrum would be produced by chance. We will consider as relevant signals those with a probability $(1-p)$ greater than $95 \%$. We calculated this probability for the global wavelet spectrum, an average in time of the power spectrum, and for the two strongest peaks (called first and second maximum) of each time slice. More details about the analysis can be found in Ugarte-Urra et al. (2004) or O'Shea et al. (2001).

We produced a light curve for each BP and each spectral window by integrating the emission coming from an area of $3 \times 3$ or $4 \times 4$ pixels, depending on the exposure time, which increases the signal to noise without loss of spatial information due to the broad point spread function of the instrument (Pauluhn et al. 1999). While for the case of the BP located at equatorial latitudes the region of integration was corrected for solar rotation, this correction was not necessary for the BPs located at the poles. The general trend of intensity increase/decrease of the light curves were subtracted from each BP with an appropriate running average in order to analyze the variations that it experiences with respect to the trend.

We describe now the results obtained for each of the BPs. The sequences with a $60 \mathrm{~s}$ cadence designed for coronal hole studies showed a saturation of the O v $629 \AA$ BP counterpart, so the study could only be carried out on He I $584 \AA$ and Mg VII/IX $368 \AA$. Special attention is given to the sequence of 30 s exposure where O v $629 \AA$ can be safely analyzed and where a comparison with high resolution MDI magnetograms is available.

\subsection{January 20 dataset}

This is one of the sequences run at polar coordinates. The BP appears in the He I $584 \AA$ window as a bright network element not very different from the surrounding area. Its emission ranges between values comparable to those of other network elements and values 35\% higher. In the $\mathrm{Mg}$ vII/IX window, the BP is clearly identified as a $20^{\prime \prime}$ round structure with an intensity between 1.5-2 times the emission of the surrounding corona. The flux lightcurve was obtained from integrating the emission of the BP central part ( $3 \times 3$ pixels). The wavelet analysis of the time-series shows an oscillatory pattern with a period of $\approx 822 \mathrm{~s}$ at the beginning of the time-series in He I $584 \AA$. It extends over 3-4 complete cycles as can be seen in the sinusoidal dashed line plotted over the detrended time-series (Fig. 1, top panel). It is preceded and followed by two brightenings of a longer temporal response, of around $1000 \mathrm{~s}$. The amplitude of the intensity variations is of the order of 5-20\%. The wavelet analysis shows that no oscillations are present above the probability threshold in the Mg vI//x time-series. The amplitude of the intensity variations $(2-6 \%)$ are much smaller than those in $\mathrm{He}$.

\subsection{January 30 dataset}

The BP, seen also in Mg viI/IX as a non-structured fuzzy bright compact region over the background coronal hole, lies over a He I network element very close and sometimes indistinguishable from the neighbouring network elements. These neighbouring elements also play an active role in the evolution of the BP. Again, as in the previous dataset, sole inspection of the He I $584 \AA$ images does not tell us which of the network elements are associated with a BP. The flux in the appropriate network element is comparable to the flux of other elements in many of the snapshots, although it can double their brightness at different times. To produce the light curve we selected a $3 \times 3$ pixel box in the central region of the $\mathrm{Mg} \mathrm{vI} / \mathrm{IX}$ image and its corresponding counterpart in the cooler line. The He r emission shows a high variability again in this case. 

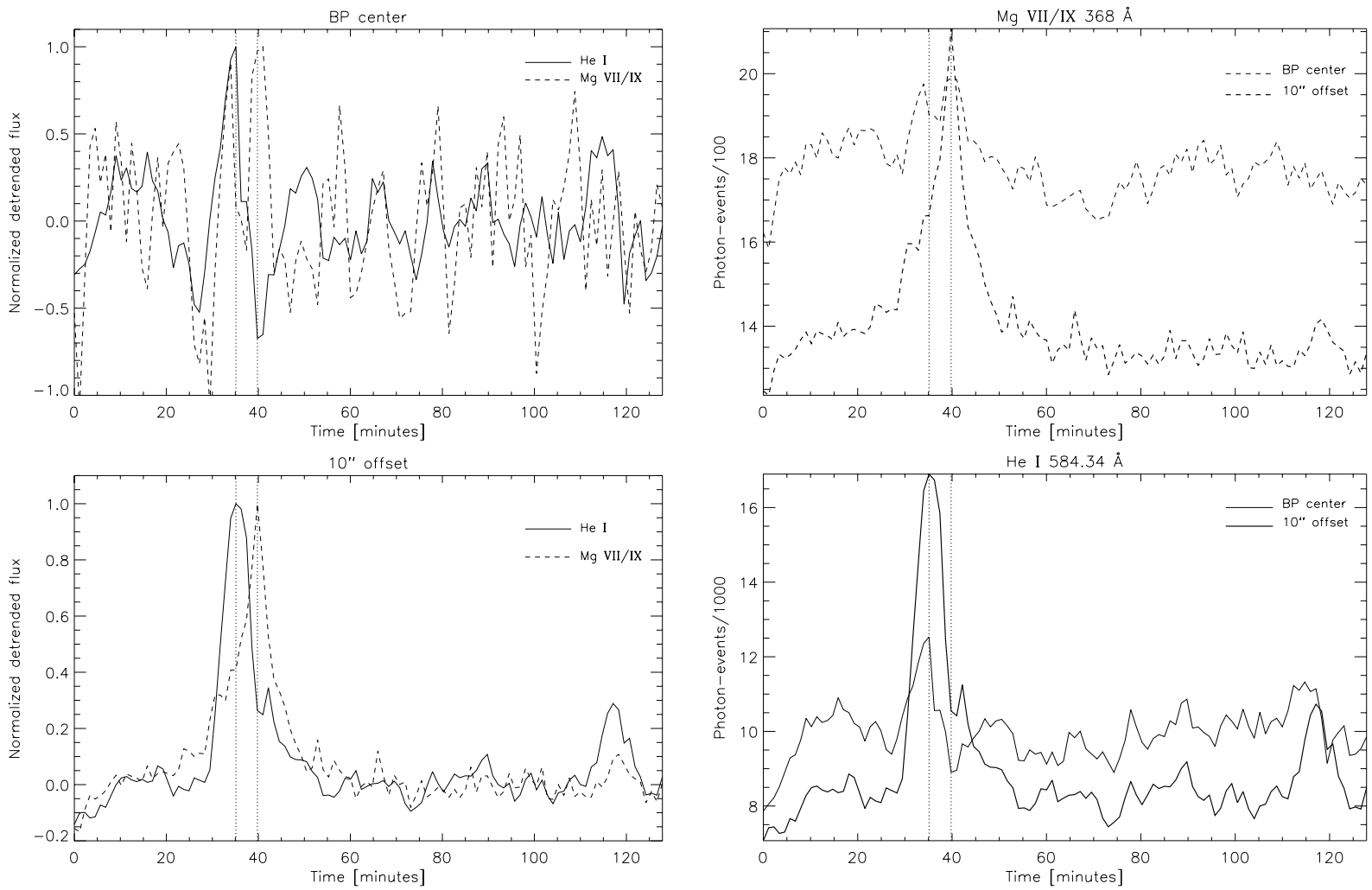

Fig. 2. He I (solid line) and Mg vi/Ix (dashed line) light curves of the BP observed on January 30. Two different locations are considered: BP center and an offset of $10^{\prime \prime}$ north of the BP center. Left panels: comparison of the temporal behaviour of the two spectral lines at the indicated location; the flux has been normalized to the maximum value of each curve to make the comparison easier. Right panels: comparison of the two locations for the same spectral line. The vertical dotted lines indicate the time when the events peaked in the off-center box.

We find intensity changes with amplitudes of the order of $4-10 \%$, slightly lower than the previous dataset, and between $3-6 \%$ for $\mathrm{Mg}$ VII/IX.

The wavelet analysis reveals the presence of a band in the power spectrum corresponding to a period of $634-754 \mathrm{~s}$ for as long as 3 cycles. The probability ranges between 80 and $96 \%$, perhaps indicative of a real oscillatory behavior, although it is below our initial probability requirements. Mg vII/IX does not show an oscillatory pattern in the power spectrum. It experiences, however, changes in the flux similar to the ones shown by He I. The top left panel of Fig. 2 shows the detrended light curve for He I (solid line) and Mg VII/IX (dashed line) normalized to their maximum values in order to make the comparison easier. Apart from the general trend, removed here, the figure shows common brightenings suggesting a connection between events at both temperatures.

There are, as well, events which are restricted to only one of the observed spectral lines, like the one which takes place in Mg VII/IX $40 \mathrm{~min}$ after the start. This is an interesting event as seen in the wide slit movie. It is originated in a nearby network element that brightens and establishes an emission link, probably loops, with the element below the coronal BP. Unfortunately, the available resolution does not allow us to establish this more firmly. The bottom left panel of Fig. 2 shows the light curve obtained from a box of integration located over the bridge between the network elements, north of the BP center. In He I, this box is located $5^{\prime \prime}$ eastwards, as the peaks of emission in He I and $\mathrm{Mg}$ VII/IX are slightly offset. The right panels of Fig. 2 show the similar information, but this time we show in each graph the light curves of the two locations for the same spectral line. The vertical dotted lines indicate the time when the events peaked in the off-center box. The large rise in the $\mathrm{He}$ I flux (34\% from trough to crest) starting at around 27 min after the beginning of the observations in the neighbouring network becomes visible in $\mathrm{Mg}$ VII/IX over the diffuse outskirts of the BP, 10-13" off-center, for just the duration of the event. The right panels show how the brightening, originated off-center in He I, produces a general rising of the coronal emission in the BP (center and offset), followed by a steeper increase, reaching $50 \%$, at the top of that bridge and peaking $281 \mathrm{~s}$ after the initial He I brightening. There is no such response in the He I emission after the first event. It soon regains its original flux, except for a little spike $143 \mathrm{~s}$ after the coronal peak. The final spike in the decay of the He I flux could be a response to the coronal emission. The formation of $\mathrm{He}_{\mathrm{I}}$ is still under discussion and one of the possible mechanisms is a reprocessing of EUV coronal radiation (Andretta et al. 2003).

\subsection{January 31 dataset}

A third BP was observed $60^{\prime \prime}$ north of the January 30 BP's location, inside a coronal hole. It appears in Mg vII/IX $368 \AA$ as a $20^{\prime \prime}$ compact bright region with a peak emission $\approx 2$ times the surrounding corona. In He I $584 \AA$ we find network 


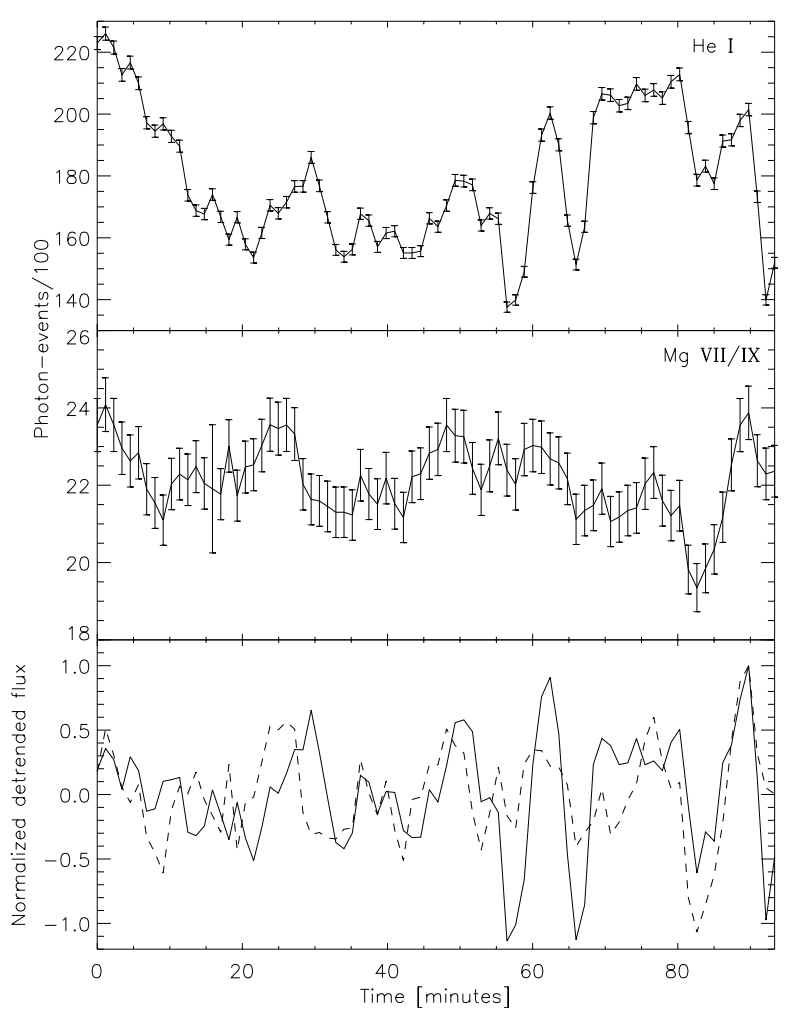

Fig. 3. He I $584 \AA$ and Mg viI/Ix $368 \AA$ flux changes observed in a BP on January 31, 1997. Top two panels: flux variations for each of the lines. Bottom panel: both light curves have been detrended and normalized to its maximum value ( $\mathrm{He} \mathrm{I}$, solid line; $\mathrm{Mg}$ vII/IX, dashed).

elements which are 1.8-2.7 times brighter than the elements with no coronal counterpart.

The length of the time-series is only $93 \mathrm{~min}$, shorter than previous datasets. The box of integration $(3 \times 3$ pixels $)$ was located in the center of the emission. The light curves obtained for both spectral lines are shown in the top two panels of Fig. 3, with the corresponding error bars obtained from the quadratic sum of the errors of individual pixels. The errors in individual pixels were obtained from the standard formulae for NIS (Thompson 2000). The bottom panel shows a comparison of the variations experienced by the BP in both lines after detrending and normalizing. These variations are very similar in both lines suggesting a connection between the events. Some of these events peak at the same time in both lines, but in other cases, like the one which peaks in $\mathrm{Mg}$ VII/IX 25 min after the start or the one peaking at minute 48 , are seen in He I with some time delay. The delay is three datapoints (204 s) in the first case and two (143 s) in the second. Brightenings with no clear counterpart in the other line are present as well. The variety of events suggests that the evolution of the flux in the BP (at this resolution) has a complex picture with different processes producing different observational patterns. The amplitudes of the variations are of the order of 5-18\% for $\mathrm{He}$ I and 3-10\% for $\mathrm{Mg}$ VII/IX.

The wavelet analysis results of the two time-series are shown in Fig. 4. The power spectrum (central panel) of both time-series has a high power band at around $700 \mathrm{~s}$. In He I, the peak is at a period of $688 \mathrm{~s}$, while in $\mathrm{Mg}$ VII/IX it is at $750 \mathrm{~s}$, with a 99-100\% confidence if we attend to the result of the wavelet analysis of the global wavelet spectrum. The time dependent plot of the probability shows, however, that certain parts of that band are below the $95 \%$ confidence level. In He I the probability is over $95 \%$ from minute 41 in the time-series. In the case of the coronal line, some points lie below the threshold, although most of the time around $95 \%$ confidence. The timeseries can be reconstructed using its wavelet power spectrum, following the indications given by Torrence \& Compo (1998). In this reconstruction, which basically sums the contributions of the different periods at the different times, we can disregard the higher periods thus revealing signals with lower periods. If we filter the periods greater than $1000 \mathrm{~s}$, the confidence level rises above the threshold. Sinusoidal functions of periods $688 \mathrm{~s}$ and $719 \mathrm{~s}$ have been plotted over the detrended light curves (top panels of Fig. 4), where $719 \mathrm{~s}$ is the middle value between the 688 and 750 values that dominate the $\mathrm{Mg}$ VII/IX power spectrum band. It stands out in the comparison that there is a regularity in the appearance of the larger brightenings suggesting that a periodic phenomenon is taking place.

\subsection{January 16 dataset}

This bright point, located at Sun center, is visible for several hours in EIT (at least from 8 UT to 14 UT) and it appears as a point structure of $3 \times 3$ pixels in the $195 \AA$ passband. Figure 5 shows the appearance of the BP in the CDS wide slit images. In Mg VII/IX $368 \AA$, we see it as a diffuse elliptical cloud with enhanced emission 2.1-2.5 times over the background. In O v $629 \AA$ we can identify between 2 and 4 network elements (depending on the instance) at the junction of three network cells. The network elements present high variable emission and their increase/decrease is not always simultaneous. He I $584 \AA$ images are very similar to the $\mathrm{O} v$ images with the network elements clearly delimiting the edges of the cells. A MDI high resolution magnetogram is also displayed in the figure and the contours of the Mg VII/IX and He I emission are over-plotted for comparison. A bipolar region associated with the coronal emission and dominated by the negative fragments is present. As we see in the $\mathrm{O} v$ and $\mathrm{He} 1$ images, there are other bright network elements in the field of view, but they do not have a coronal counterpart in $\mathrm{Mg}$ VII/IX. It is easy to notice that the coronal emission is associated with the presence of strong opposite polarities. The positive fragments associated with the BP and the surrounding bright area have peaks in the magnetic flux density between 97 and $187 \mathrm{Mx} \mathrm{cm}^{-2}$, while the positive fragment seen at an offset of $\left(15^{\prime \prime}, 65^{\prime \prime}\right)$ (associated with the bright $\mathrm{O} v$ network element) does not reach $40 \mathrm{Mx} \mathrm{cm}^{-2}$.

Due to a higher cadence than previous sequences (31 s), we decided to use a larger box of integration, $4 \times 4$ pixels, to increase the signal to noise. This box was located at the center of the peak emission in Mg VII/IX. That same position in the He I image corresponds to the left network element below the BP in Fig. 5 and the same occurs for the $\mathrm{O} v$ image, where in this instance the network element is smaller than the neighbouring elements at the network junction. A first glance to the time-series of each of the spectral lines indicates that the 

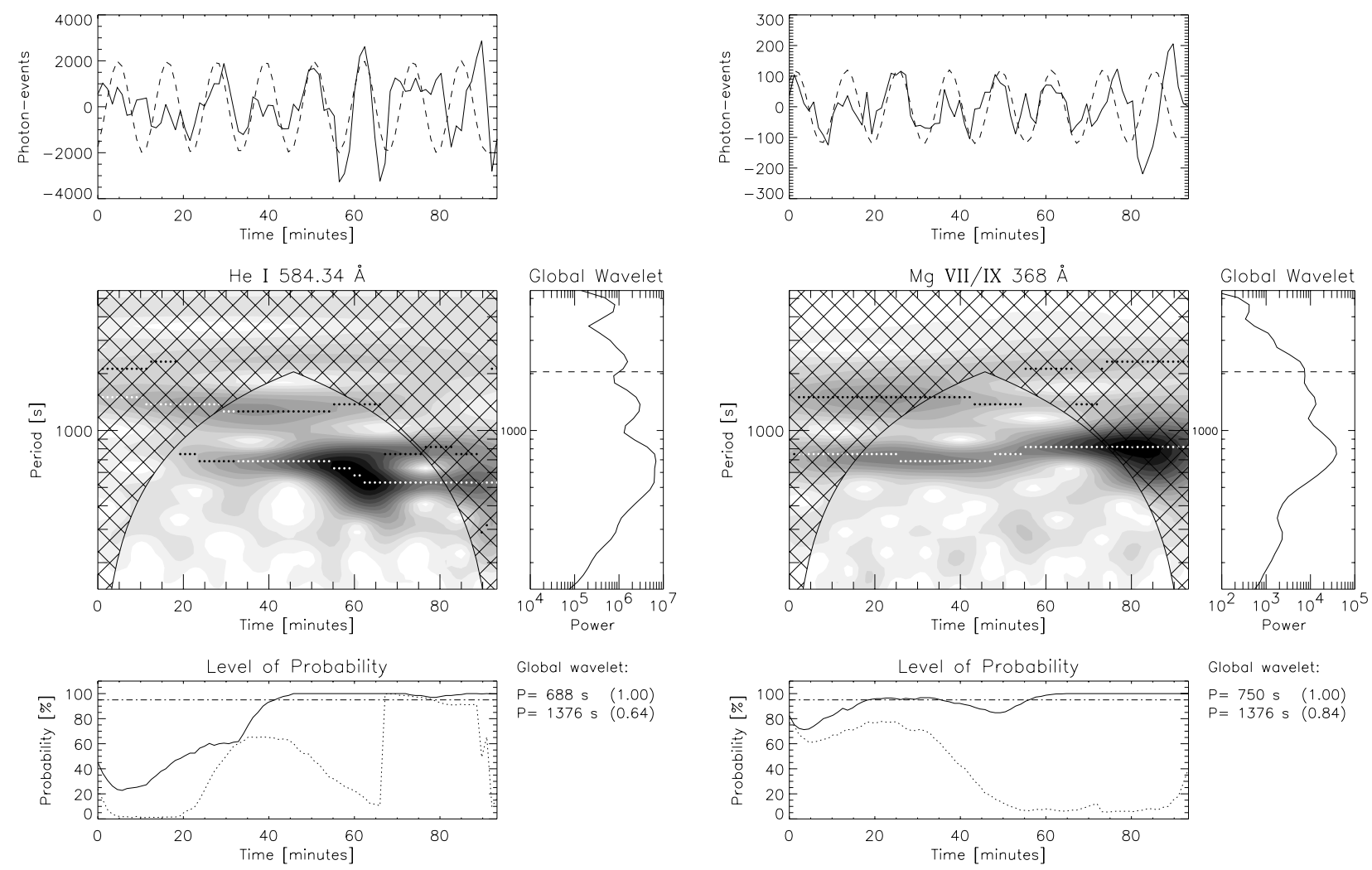

Global wavelet:

$P=750 \mathrm{~s} \quad(1.00)$

$=1376 \mathrm{~s}(0.84)$

Fig. 4. Wavelet analysis results of the BP observed on January 31, 1997 at 08:02 UT. The left panel corresponds to the He I $584.34 \AA$ time-series and the right panel to Mg vII/Ix $368 \AA$. The dashed lines plotted over the two light curves at the top panels account for sinusoidal functions with periods of 688 and $719 \mathrm{~s}$ respectively, corresponding to the peaks of the power spectrum.
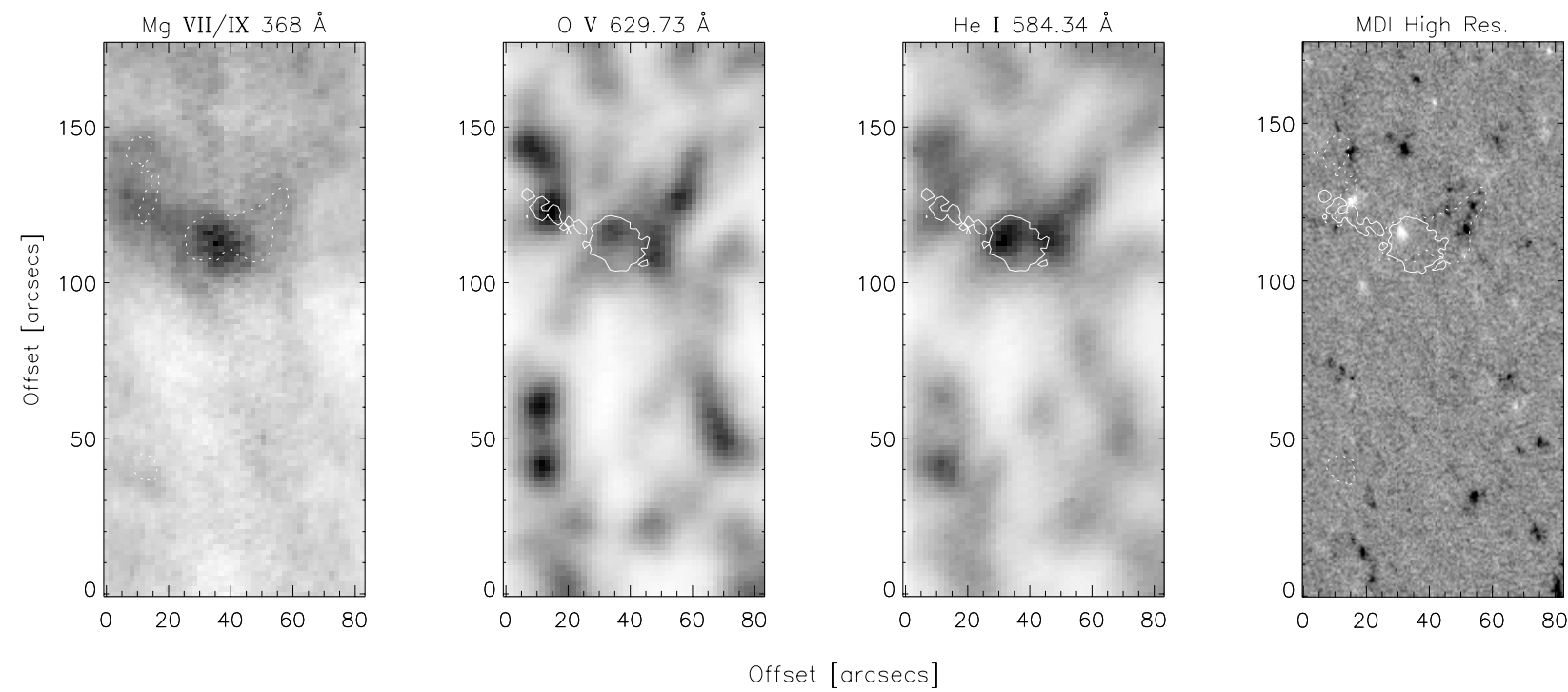

Fig. 5. CDS wide slit images in Mg vII/Ix $368 \AA$, O v $629.73 \AA$ and He I $584.34 \AA$, of the coronal bright point observed on January 16,1997 , plus a MDI high resolution magnetogram. In the images, black regions are the brightest sources and white the faintest. In the magnetogram, positive polarities are in white and negative in black. All images were taken at 10:01 UT. Solid (Mg vII/IX) and dotted (He I) contours are used as a reference for comparison.

general BP emission increases monotonically all along the observation period and that the variability is higher as the emission increases. This general trend has been removed, using an appropriate running average for each of the light curves, in order to study the variations with respect to the trend. The amplitude of these variations are of the order of $3-11 \%$ in the case of the He $1584 \AA$ emission, 10-20\% in the case of O v $629 \AA$ with events reaching up to $45 \%$, and $3-8 \%$ for Mg VII/IX $368 \AA$.

The wavelet analysis of the He I light curve (top left panel of Fig. 6) reveals a diagonal band in the power spectrum, 97 min long over the $95 \%$ confidence level, decreasing from a period of $896 \mathrm{~s}$ to a period of $257 \mathrm{~s}$ in the second half of 

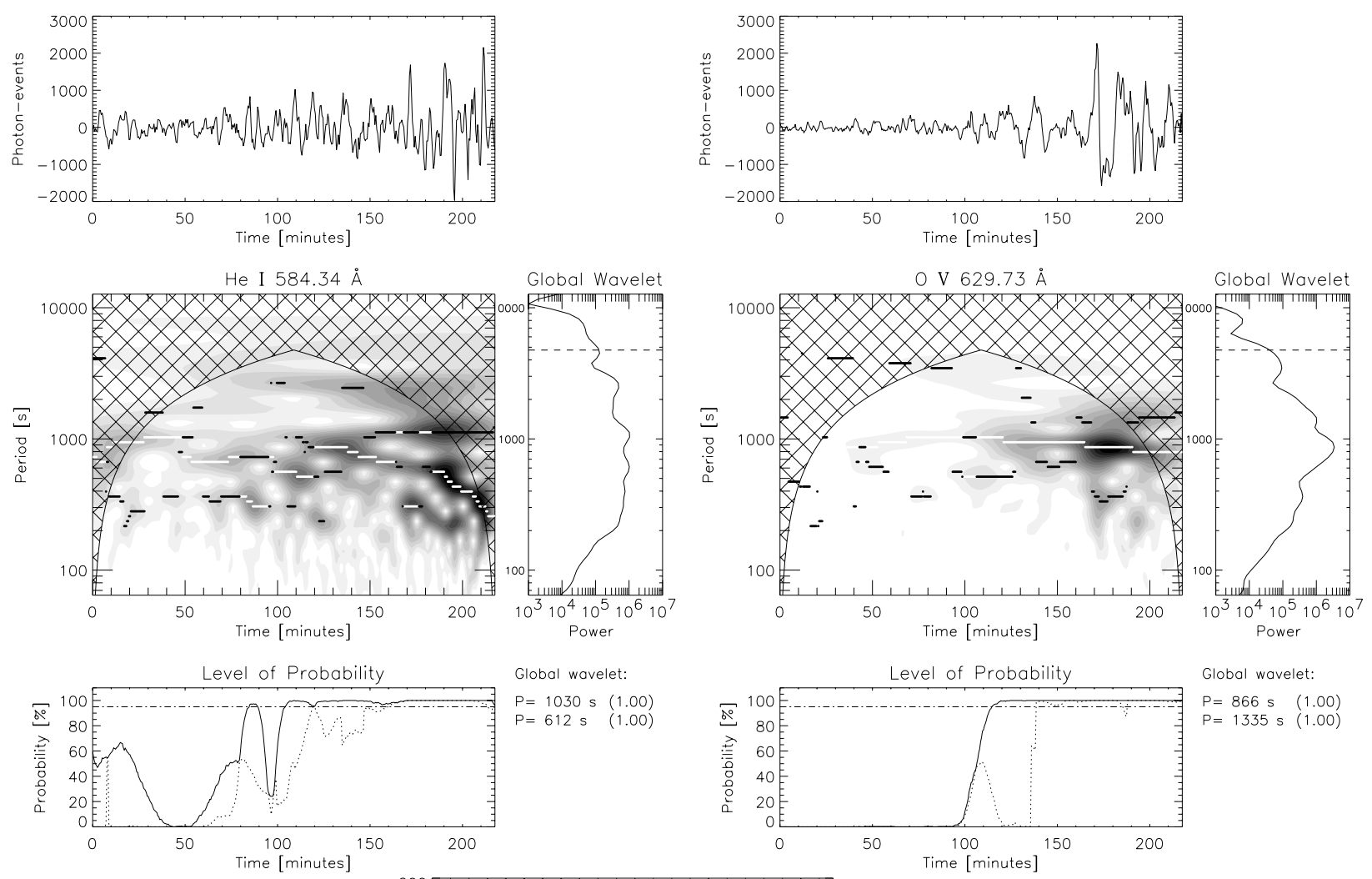

Global wavelet:
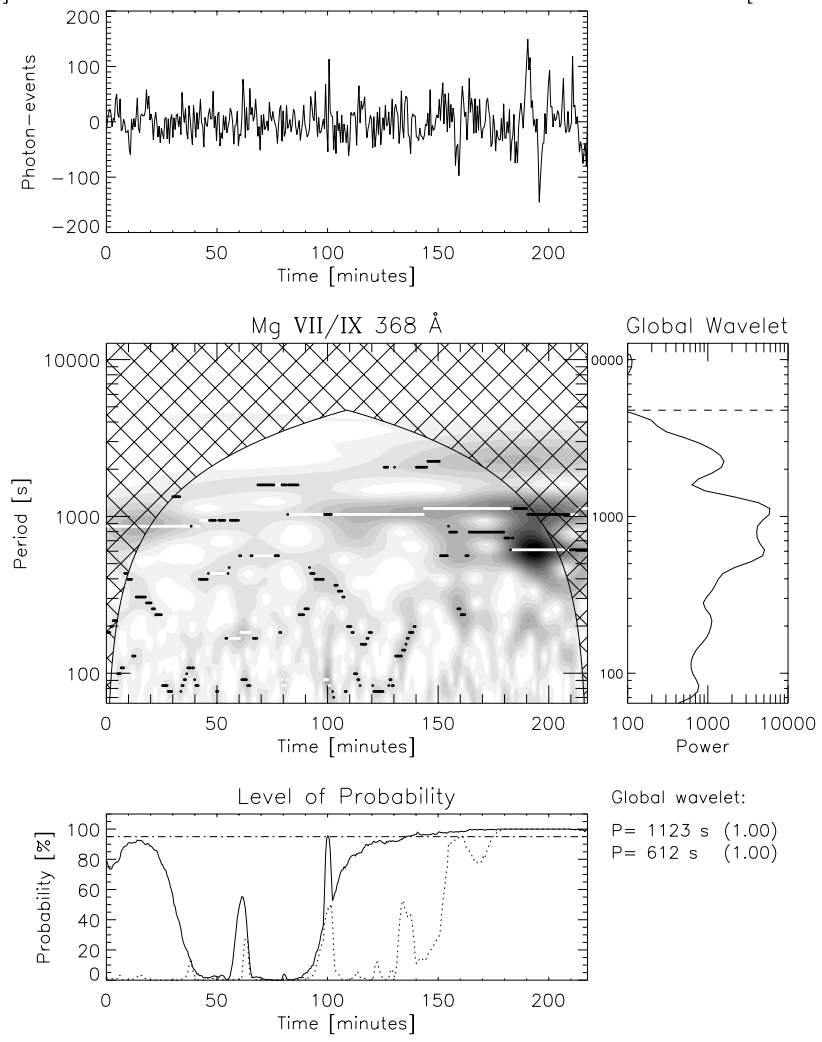

Global wovelet: $\begin{array}{ll}P=1123 s & (1.00) \\ P=612 s & (1.00)\end{array}$

Fig. 6. Wavelet analysis results of the BP observed on January 16, 1997 at 08:02 UT. Top left panel corresponds to the He I $584.34 \AA$ time-series, top right to the O v $629.73 \AA$ A ones and bottom to the Mg viI/Ix $368 \AA$ blend.

the time series, but it does not last for more than two cycles in any of the periods. There is, however, a peak in the power spectrum at around $1123 \mathrm{~s}$ that lasts for $63 \mathrm{~min}$, with the first two cycles well outside the cone of influence. Simultaneous to that we can identify a band with short periods (306-236 s), two and four cycles long each. We checked that the running average is not introducing false frequencies by removing the trend and longer periods in a different way. We applied the wavelet 

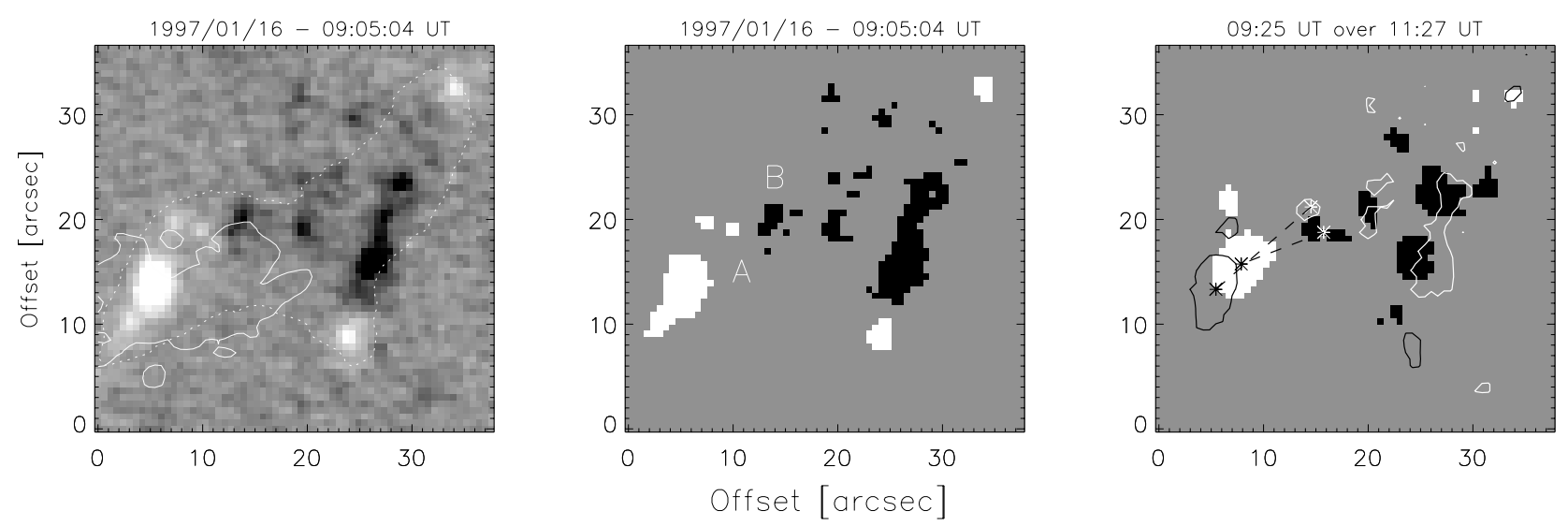

Fig. 7. Section of a five minute averaged MDI high resolution magnetogram showing the photospheric magnetic configuration associated to the Mg VII/IX BP observed on January 16, 1997. On the left panel, the solid and dotted contours locate the Mg viI/IX and O v emission, respectively. The section is a close-up of the region centered at approx. (45", 120") in Fig. 5. White accounts for positive magnetic flux density, $\phi$, and black for negative. In the center and right panels we plot only those pixels with $|\phi| \geq 21 \mathrm{Mx} \mathrm{cm}^{-2}$. The right panel shows the maximum change in orientation $\left(\sim 20^{\circ}\right)$ of the straight line joining the centroid positions of the two opposite polarities for two different images. White/black contours correspond in this case to the negative/positive polarity.

analysis to the un-detrended light curve and reconstructed it using the power spectrum with only the shorter periods taken into consideration. We find similar results, which give us confidence that the peaks in the power spectrum have a solar origin.

The analysis of the $\mathrm{O} v$ light curve shows a power spectrum with certain similarities to He I. A band with a confidence level over the 95\%, with periods ranging between 944 and 794 s, appears at the same time as the diagonal band in He I, suggesting a connection between the events. In this case, the peak in the power spectrum at $944 \mathrm{~s}$ can host around 3 oscillatory cycles. The same occurs for the peak at 364 s simultaneous to the one found for He I at $306 \mathrm{~s}$.

In the case of $\mathrm{Mg}$ VII/IX we can clearly identify, at the bottom panel of Fig. 6, the band peaking at $1123 \mathrm{~s}$ that we found in the He I data. It is long enough to host 4 complete cycles, 3 of them outside the cone of influence. The shorter periods, $216 \mathrm{~s}$, barely seen in the figure can also be detected in this case using the reconstruction technique, but only marginally and for less than two cycles over the $95 \%$ confidence level. Somehow, the fact that they are present at the same location as in the cooler lines suggests that they could be real and therefore could be part of the coronal evolution of the BP. The lower signal to noise of this spectral line and the mixture of different structures with the current spatial resolution are all possible causes that this oscillation is partially hidden in Mg VII/Ix. Finally there is another period peaking at $612 \mathrm{~s}$, close to the end of the temporal sequence.

\section{Magnetic field evolution}

It was noted in the introductory section the importance of the evolution of the magnetic fragments and the associated magnetic flux in relation to the BP appearance (see also Ugarte-Urra et al. 2004). We feel that the present discussion will benefit if we discuss the EUV brightness fluctuations in the context of the changes experienced by the magnetic field.
We do it, therefore, for one of the BPs for which we have MDI high resolution magnetograms.

The MDI high resolution magnetograms $\left(0.6^{\prime \prime} / \mathrm{pixel}\right)$ are available for the BP observed on January 16 . The observations cover the whole CDS observing time with a cadence of one magnetogram every minute. In order to improve the signal to noise we produced new magnetograms by averaging five consecutive images, which left us a final cadence of five minutes. The noise in the dataset can be obtained from the core of the distribution function of flux densities, $\phi$ (Hagenaar 2001). This core is well described by a Gaussian fit, which reveals in our case a level of noise of $\sigma=11.1 \mathrm{Mx} \mathrm{cm}^{-2}$.

The magnetic configuration of the $\mathrm{BP}$ area is visible in Fig. 5 (right panel). The left image in Fig. 7 shows a close-up of the region centered approximately at the offset coordinates of $\left(45^{\prime \prime}, 120^{\prime \prime}\right)$ in Fig. 5, with the Mg VII/IX and O v isocontours to make the comparison easier. The alignment between CDS and MDI was done using the header coordinates. The uncertainty is not larger than $5^{\prime \prime}$. There is a main negative polarity made of several fragments that dominates and three satellite positive polarities around it, one of them stronger than the others, see $\mathrm{A}$ in Fig. 7. The corresponding $\mathrm{O} v$ and $\mathrm{He}_{\mathrm{I}}$ images show three network elements associated with the three points where opposite polarities are confronted. Attending to the evolution of these satellite polarities we find in the images from 2 to 4 network elements associated with the coronal emission, which mainly lies over the left interaction point, A. The movie, made with the collection of images, shows an approaching movement of the two polarities, A and B, at this location, as well as a disappearance or cancellation of the lower positive fragment.

We wanted to inspect this issue in order to see the influence of the magnetic evolution on the EUV emission, which becomes more variable and with oscillations more evident in the last part of the observing period. Following the method described by Parnell (2002), we calculated the survival functions of the magnetic flux density distribution and the Gaussian fit representing the noise. The survival function gives the 


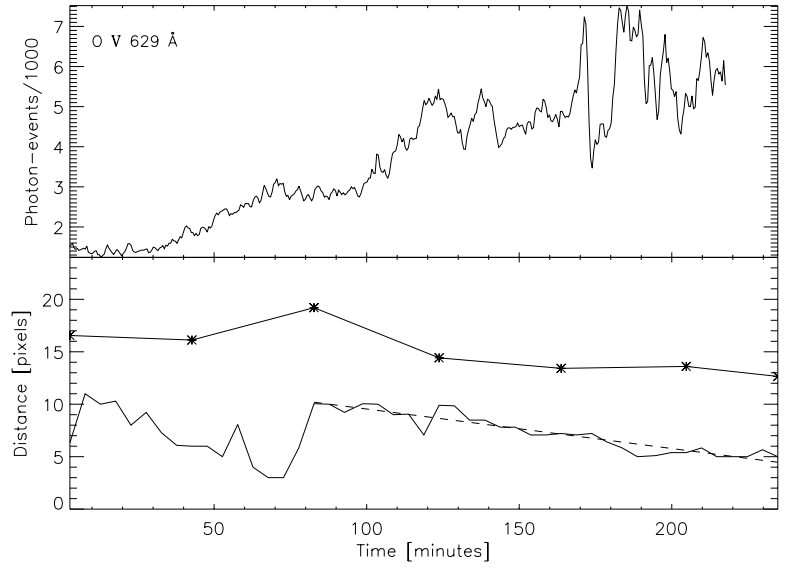

Fig. 8. Comparison between the temporal variation in the transition region emission of O v $629.73 \AA$ (top) and the distance between opposite polarities A and B (bottom). The asterisks account for the distance between centroids and the single solid line represents the distance between the closest pixels. The dashed line is a linear fit to the second distance decrease section used to determine the approaching velocity of $0.27 \mathrm{~km} \mathrm{~s}^{-1}$.

probability for a certain variate to be greater than a certain value. In this case the variate is the magnetic flux density. From the ratio of the two survival functions we obtained the proportion of pixels associated with noise for the different flux density values. At $21 \mathrm{Mx} \mathrm{cm}^{-2}$ less than $1 \%$ of the pixels are associated with noise. We adopt this as our working value. We plot in the center and right panels of Fig. 7 the BP magnetic configuration considering only those pixels with $|\phi| \geq 21 \mathrm{Mx} \mathrm{cm}^{-2}$.

We determined then the distance between $\mathrm{A}$ and $\mathrm{B}$ all along the time-series in two ways. First of all, we calculated the distance between the two closest pixels with a magnetic flux that we know for certain it is not associated with noise, i.e. those pixels with $|\phi| \geq 21 \mathrm{Mx} \mathrm{cm}^{-2}$. Secondly, we calculated the distance between the centroids of both polarities at different instances of the time-series. Both results are shown in Fig. 8, together with the temporal changes in the number of counts (photon-events) in the transition region line. Asterisks represent centroid distances, while the single solid line accounts for the distance between closest pixels. The approaching trend is clear in both curves. The latter shows two different sections of decreasing distance joined by a steep increase at around $80 \mathrm{~min}$ after the start of the observing period. This increase is due to the cancellation of the positive fragments that we see detached from the main positive polarity in the left and center panels of Fig. 7. After the disappearance of these fragments, the closest pixel belongs to the largest positive fragment in the figure, which is further apart. The separation between centroids shows a similar profile due to the same phenomenon. This is the distance between the strongest flux concentrations since we only consider pixels with a flux over $21 \mathrm{Mx} \mathrm{cm}^{-2}$. The real distance between opposite polarity fragments would probably be less than that. A comparison with the CDS emission reveals that the approach of the polarities is followed by an increase in the transition region flux. Interestingly, the flux increase reaches a 30 min "plateau" when the distance between polarities experiences the steep increase, and as soon as it starts to decrease

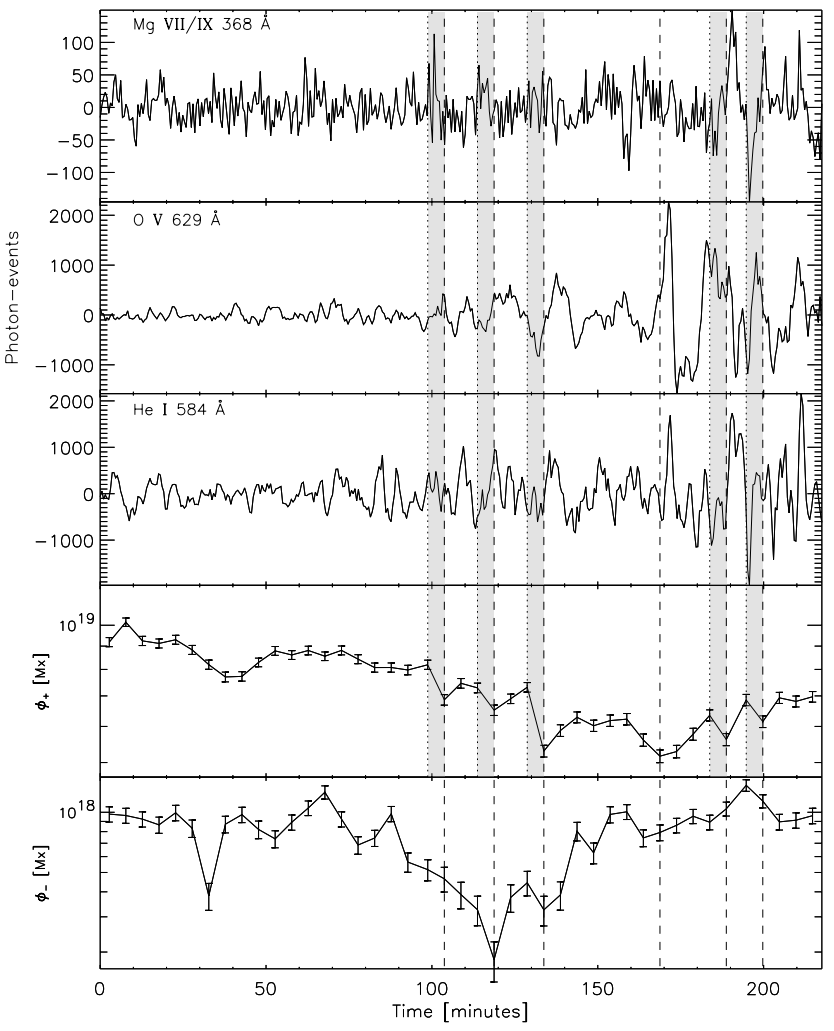

Fig. 9. Comparison between the EUV variability of the BP detrended emission, as obtained from the CDS wide slit images, and the magnetic flux of the two opposite polarities. Shaded areas point out locations where we see a decrease in the positive flux preceding a brightening in the EUV lines. The local minimum is given by a dashed line.

again, the flux rises. Another characteristic is that the amplitude of the $\mathrm{O} v$ brightenings becomes larger with time. The fact that the closest distance between polarities is reached just before the "plateau" phase and the amplitudes are not the largest at this moment, suggests that the proximity of the centroid, or in other terms the bulk of the positive magnetic flux, is more important in terms of energy release. From these plots we can infer the approach velocity. From a linear fit (Fig. 8) to the second section of the curve that gives the distance between closest pixels (i.e. when the small fragments have already been canceled), we find a velocity of approach of $0.27 \pm 0.02 \mathrm{~km} \mathrm{~s}^{-1}$. In the case of the centroids, we obtain a value of $0.15 \pm 0.06 \mathrm{~km} \mathrm{~s}^{-1}$ using all the points. If we just fit the points after the small fragments have canceled the result is $0.26 \pm 0.09 \mathrm{~km} \mathrm{~s}^{-1}$. These results are inside the range of typical values $\left(0.1-0.3 \mathrm{~km} \mathrm{~s}^{-1}\right)$ quoted by Priest et al. (1994).

Once a reasonable threshold has been chosen to delimit the polarities, what we want to do is to compare the evolution of the emission in the different spectral lines with the evolution of the integrated magnetic flux of the polarities. This comparison is shown in Fig. 9. The top three panels represent the BP's detrended flux variations in $\mathrm{Mg}$ vII/IX, $\mathrm{O}$ v and $\mathrm{He}$. The bottom two panels show the evolution of the positive magnetic flux in $\mathrm{A}, \phi_{+}$, and the negative magnetic flux in $\mathrm{B}, \phi_{-}$. The two values are obtained from the integration for each image of those pixels with $|\phi| \geq 21 \mathrm{Mx} \mathrm{cm}^{-2}$ in the region containing A 
and B. From this comparison the first thing that we notice is that the monotonic increase of the EUV emission, visible in Fig. 8 for $\mathrm{O} v$ and similar for the other two lines, is associated in Fig. 9 with a decrease in the positive magnetic flux, which is an order of magnitude larger than the negative one. On smaller timescales, the plot suggests that sudden decreases in the positive magnetic flux (shaded areas) seem to take place just before (or during the rising phase) a brightening is observed in the transition region or the corona, and sometimes both. The location of the local minimum in the magnetic flux is given by a dashed line. These results could suggest that magnetic flux cancellation are associated to the stronger brightenings and give support to the picture that proposes reconnection as the main process producing the appearance of BPs and its characteristic variability (Priest et al. 1994; Longcope 1998). However, we feel that more observations are needed to confirm a definite correspondence between decreases in the magnetic flux of this order and the brightenings. The negative flux does not show as good correlation as the positive one, but this could be explained by the fact that fragment $\mathrm{B}$ is weaker in flux and also belongs to a more complex structure, where the surrounding positive fragments (see Fig. 7) could play an active role in the evolution.

\section{Discussion}

It is clear from the present work and that of Madjarska et al. (2003) and Ugarte-Urra et al. (2004) that BPs have intermittent (few cycles), but clearly defined periods of oscillation ranging from around $400 \mathrm{~s}$ up to $1100 \mathrm{~s}(0.9-2.5 \mathrm{mHz})$ in upper chromospheric, transition region and sometimes coronal plasma. In the case of one BP, periods as short as $236 \mathrm{~s}(4.2 \mathrm{mHz})$ were also detected. Apart from the clear oscillatory cases, we have found several other cases with similar periods where the probability estimates do not reach the required $95 \%$, but they are very close. We believe that some of these oscillations are real, in particular those detected simultaneously in several spectral lines, but are hidden in the low spatial resolution of the integrated emission.

Oscillations in the quiet Sun chromosphere have been known for a long time and is a well documented phenomenon. Two regions showing two different characteristic frequency ranges are normally differentiated. On one side the $\sim 5.5 \mathrm{mHz}$ (3 min) oscillations seen in the chromospheric internetwork (Dame et al. 1984; Carlsson et al. 1997; Curdt \& Heinzel 1998; Krijger et al. 2001; Wilhelm \& Kalkofen 2003, and references therein); on the other, the network lanes and the network bright points (NBPs), which show a wider range of frequencies $(0.8-3.3 \mathrm{mHz}), 5-20 \mathrm{~min}$ periods according to Lites et al. (1993; but see also Dame et al. 1984; Curdt \& Heinzel 1998; Doyle et al. 1999; Banerjee et al. 2001; McAteer et al. 2002, 2003). The four BPs analyzed here lie on the network lanes and their $\mathrm{O} v$ and $\mathrm{He}$ I counterparts are network elements generally indistinguishable from other elements with no coronal counterpart. The first thing that we notice is that the frequencies found for these BPs lie in the frequency range of the chromospheric network oscillations. One characteristic of NBPs is that they are associated with photospheric magnetic flux elements (Cauzzi et al. 2000). This can be clearly seen in Fig. 2 of McAteer et al. (2003), which shows the appearance of several NBPs at different temperatures in the solar atmosphere, from the chromosphere, through the transition region to the corona, co-aligned with a magnetogram. It is worth noting that McAteer et al. (2003) NBP 5 shows the characteristic size and enhanced coronal emission overlying two opposite polarity magnetic fragments of a coronal bright point (with a peak emission around 2 times higher than the surrounding corona, as checked from the original image). In the transition region image we see two bright features at the location where the footpoints of the coronal loop would be, connected by what looks like a cooler loop structure; these footpoints can be traced into the chromosphere and the two opposite polarities in the magnetogram. Only the brightest one was the subject of the NBP study by the authors. Figure 2 in Cauzzi et al. (2000) shows a similar configuration with two opposite magnetic fragments of reasonable strength for a coronal bright point, separated by less than $10^{\prime \prime}$, below the chromospheric emission of two NBPs. No coronal image is available in this case. More images can be compared in Nindos \& Zirin (1998).

This leads us to the conclusion that a fraction of the NBPs (and the associated magnetic field) are most likely the footpoints of the tiny loops which comprise coronal bright points (Sheeley \& Golub 1979); the cool footpoints of loops which rise to greater temperatures (Cook et al. 1983). This would explain why we see the same periodicities in both features. Recently, McAteer et al. (2004), analyzing quiet Sun network and internetwork TRACE oscillations, found that the network has a peak occurrence rate periodicity of 231-346 s, with a significant tail of higher period oscillations (as long as $\sim 1000 \mathrm{~s}$ ). The peak decreases in occurrence rate in the highest formation temperature passband $\left(\leq 1 \times 10^{5} \mathrm{~K}\right)$ suggesting that the waves may dissipate, shock, move away from the network or change frequency. Gouttebroze et al. (1999) did not find signature of $2.5-7 \mathrm{mHz}$ (period of 143-400 s) oscillations in lines with a temperature formation $\geq 5 \times 10^{4} \mathrm{~K}$. If we bear in mind that BP's studies (Ugarte-Urra et al. 2004, and the present one) show oscillations with typical periods of around 400-1100 s, with only one case showing shorter periods, all this seems to be consistent with a picture where only the longer periods observed in the chromospheric NBP, assumed here as the footpoints of hotter loops, make it through to the transition region and corona. However, more observations with simultaneous chromospheric and coronal counterparts of the BP will be needed to confirm that.

Different interpretations are given for these long frequency oscillations in the chromospheric network, which show no sign of distinctive peaks in the power spectrum (Krijger et al. 2001); namely, in terms of formation of standing waves by internal gravity waves (Deubner \& Fleck 1990; Lou 1995) or upward propagating MHD waves excited by granular motions (Kalkofen 1997; Hasan \& Kalkofen 1999), although more discussion is still needed (Musielak \& Ulmschneider 2003).

The modulation seen in Ugarte-Urra et al. (2004) and our results is more pronounced at lower layers of the atmosphere: in the emission lines associated with the temperatures $30000 \mathrm{~K}, 250000 \mathrm{~K}$ and $1000000 \mathrm{~K}$ (the corresponding sound speeds are $27 \mathrm{~km} \mathrm{~s}^{-1}, 76 \mathrm{~km} \mathrm{~s}^{-1}$ and $152 \mathrm{~km} \mathrm{~s}^{-1}$, respectively). 
Although we should note that the formation of $\mathrm{He}$ I line is still under discussion (Andretta et al. 2003). We see periods of around $600 \mathrm{~s}$ in $\mathrm{O} \mathrm{v}$ and $\mathrm{He} \mathrm{I}$ overlapping. The January $16 \mathrm{BP}$ is the clearest case with certain periods shared by two of the three lines in the sample ( $\mathrm{He} \mathrm{I}, \mathrm{O} v$ and $\mathrm{Mg}$ VII/IX). How are these oscillations related to the heating of BPs and how do they merge with the idea that reconnection plays a dominant role in the evolution of BPs?

One idea is that the observed intensity oscillations are associated with compressible waves, e.g. the acoustic mode which has been identified in coronal structures as both a propagating wave (e.g. Nakariakov et al. 2000) and standing waves (e.g. Ofman \& Wang 2002) or the fast mode. An alternative interpretation, as a kink mode observed through the modulation of the observed width of the structure guiding the wave (Cooper et al. 2003), may be excluded from consideration as it is not likely that the kink mode, practically incompressible, has a noticeable amplitude in the chromosphere and the transition region.

The mode oscillates between the footpoints of the magnetic field line. Along the field line, the sound speed changes, but for the global mode (or any other lower harmonics) it is the average sound speed which determines the period, not the local one, hence this would mean that the oscillations have a collective nature, e.g., similar to the global acoustic mode of a coronal loop (Ofman \& Wang 2002) or their second harmonics (Nakariakov et al. 2004). The common feature of these oscillations is the positioning of nodes of the velocity perturbations and maxima of density perturbations at the lower layers of the atmosphere. In particular, in the structure of the global acoustic mode is

$V_{z}(s, t) \propto \cos \left(\frac{\pi C_{\mathrm{s}}}{L} t\right) \cos \left(\frac{\pi}{L} s\right)$

$\rho(s, t) \propto \sin \left(\frac{\pi C_{\mathrm{s}}}{L} t\right) \sin \left(\frac{\pi}{L} s\right)$,

where $C_{\mathrm{s}}$ is a speed of sound, $L$ the loop length, and $s$ is a distance along the loop with the zero at the loop top. According to Eqs. (1) and (2), the oscillation period is given by the expression $2 L / C_{\mathrm{s}}$. As the magnetic field in a coronal bright point is closed, it may be considered as a small loop with the oscillation modes described by these equations. On the other-hand, if the magnetic topology of the bright point is more complicated than the loop geometry, its "building elements" may be modelled as individual loops. In addition, as the acoustic wave considered here propagated strictly along the magnetic field, it does not feel the magnetic field complexity, simply following the local line.

Suppose the length of the loop forming an oscillating bright point is $30 \mathrm{Mm}$ (corresponding to a $10 \mathrm{Mm}$ radius), the global acoustic mode with periods of about $600 \mathrm{~s}$ would require the sound speed to be $100 \mathrm{~km} \mathrm{~s}^{-1}$. This value of the sound speed is certainly reasonable. The period of the second harmonics of this loop is $300 \mathrm{~s}$, also in the observed range. Different periods can be easily explained by different loop lengths and by slightly different profiles of the sound speed along the loop (growing from the chromospheric $30 \mathrm{~km} \mathrm{~s}^{-1}$ to coronal $150 \mathrm{~km} \mathrm{~s}^{-1}$ ). Also, a lower lying loop would in general have lower sound speed.
The domination of the oscillations in chromospheric and TR emission lines, is consistent with this interpretation too: according to Eq. (2) the density perturbations are stronger near the loop footpoints. At the coronal heights, the oscillations are not so pronounced because the density oscillations have a node at the loop apex.

Another possible interpretation of these oscillations is that they are connected with vertically propagating fast magnetoacoustic waves trapped between the dense chromosphere and the upper boundary of the bright point magnetic structure, where the Alfvén speed experiences a sharp increase. The wavelength of such waves is about double the radius of the closed magnetic field lines, and the characteristic speed is several sound speeds (the lowest possible value is one and a half sound speed, in the case of $\beta=1$ ). Taking the values from the previous example, we obtain the longest characteristic period of about $130 \mathrm{~s}$ (for the radius $10 \mathrm{Mm}$ and the sound speed $100 \mathrm{~km} \mathrm{~s}^{-1}$ ). This value is less consistent with the observational findings than the periods given by the acoustic model.

This simple model then suggests that the bright point oscillations could be associated with the global acoustic modes of closed magnetic structures associated with the bright point. However, this does not address the important question of how these waves are generated. They could be related to the supergranular motions or may be excited by the energy deposition from the reconnection process. This second scenario would be supported by the fact that, in the case of the BP observed on January 16, we detect sudden decreases in the magnetic flux at the same time as the oscillations in the EUV flux take place.

If reconnection is important in BPs (as commonly assumed) it has to be a slow process as the observed Doppler shift of transition region lines is at most $10 \mathrm{~km} \mathrm{~s}^{-1}$ red-shifted (Madjarska et al. 2003; Xia et al. 2003; Popescu et al. 2004) compared to a red-shift of 10-25 $\mathrm{km} \mathrm{s}^{-1}$ for blinkers (Madjarska \& Doyle 2003) and \pm 100 to $200 \mathrm{~km} \mathrm{~s}^{-1}$ (Madjarska \& Doyle 2002) for bi-directional jets. It is more in the order of the quiet Sun network (Teriaca et al. 1999) which has a red-shift of $8-10 \mathrm{~km} \mathrm{~s}^{-1}$ at these temperatures.

In this respect we may have a problem. As shown in Sect. 4, the converging speed of the two polarities was measured as $0.27 \mathrm{~km} \mathrm{~s}^{-1}$ which is an excellent agreement with a study by Chae et al. (2002a) who measured the approach for two different observations of converging polarities. In one instance the speed was $0.35 \mathrm{~km} \mathrm{~s}^{-1}$, while the other was $0.27 \mathrm{~km} \mathrm{~s}^{-1}$. In follow-up work, Chae et al. (2002b) considered two reconnection models, the Sweet-Parker model and the faster reconnection Petschek model. They showed that the Sweet-Parker model does not have inflow speeds greater than $0.08 \mathrm{~km} \mathrm{~s}^{-1}$ while in the Petschek model, the inflow speed equals the observed speed just below the temperature minimum. Higher up in the atmosphere, e.g. the chromosphere and transition region, the speeds are in the range 20 to $100 \mathrm{~km} \mathrm{~s}^{-1}$. Thus, although the Petschek looks applicable around the temperature minimum it is clearly not correct in the upper atmosphere as these velocities are much greater than the maximum observed Doppler shifts. In fact, these rates would be more applicable to bi-directional jets. One possible way of resolving the Sweet-Parker rates in the lower atmosphere is to change some of the assumptions 


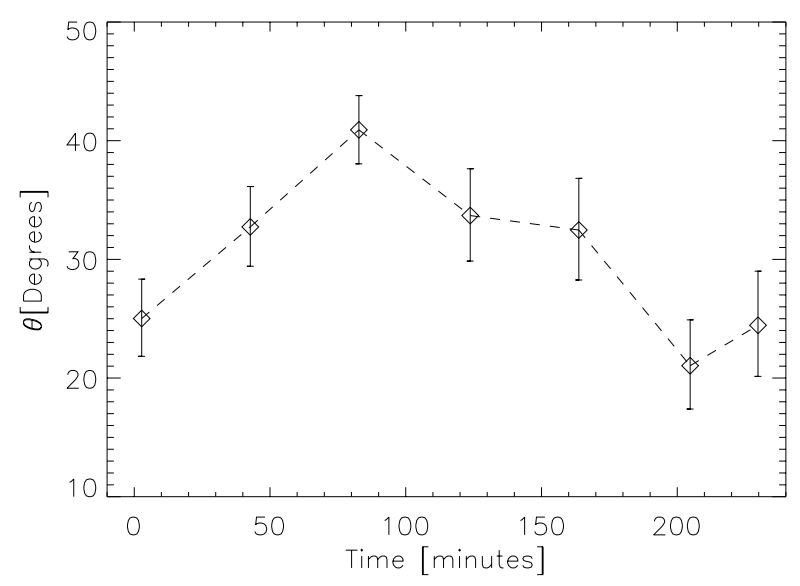

Fig. 10. Variation in time of the angle between the line joining the centroids of the two polarities of the January $16 \mathrm{BP}$ and Solar_Y $=0$.

in the model. For example, taking a very small magnetic filling factor $(\sim 0.05)$ and a small electric conductivity of $10^{10} \mathrm{~s}^{-1}$ yields an inflow speed of $0.27 \mathrm{~km} \mathrm{~s}^{-1}$ which would be consistent with the observations (Chae et al. 2002a,b). However, even the Sweet-Parker model gives large velocities in the upper atmosphere. Thus this would rule out both of these as possible reconnection models for BPs.

Binary reconnection, an interaction of pairs of unbalanced sources has been proposed (Priest et al. 2003) as a fundamental mechanism to produce coronal heating. One aspect of this mechanism is that the relative motions of the sources are likely to build-up twisted force-free structures that relax by turbulent reconnection resulting in heating. We have tested this possible scenario against the BP observed on January 16. The sources show a relative motion of approach, as described earlier, but also of rotation (right panel of Fig. 7). We have measured the angle that the line joining the two centroids makes with the Solar_Y $=0$ (Fig. 10). The figure shows how one source rotates with respect to the other reaching a maximum of $41^{\circ}$ and then returning to the original configuration. The error bars come from the uncertainty in the determination of the centroid, assumed as 1 pixel. The energy injected by this rotation can be determined using $\Delta E=F^{2} \Delta \theta^{2} /\left(24 \pi^{2} \mu L_{\text {eff }}\right.$ ) (Priest et al. 2003) with $F$ the magnetic flux of the weakest polarity, $\Delta \theta$ the angle change, $\mu$ the magnetic permeability and $L_{\mathrm{eff}}$ the effective length of the magnetic field lines. The maximum change in orientation is $\Delta \theta \sim 20^{\circ}$ and takes $122 \mathrm{~min}$. Choosing $L_{\mathrm{eff}}$ as 10.2 Mm (15 MDI pixels for the footpoint separation and semicircular loop shape) and a range of magnetic flux values ranging from $7.8 \times 10^{17}$ to $7.2 \times 10^{18} \mathrm{Mx}$ (that depend on the threshold of integration) for the weakest polarity, we obtain an energy injection per second of $\approx 10^{18}-10^{20} \mathrm{erg} \mathrm{s}^{-1}$, much smaller than the typical values $\left(10^{23}-10^{24} \mathrm{erg} \mathrm{s}^{-1}\right)$ of energy loss in BPs (Habbal \& Withbroe 1981; Priest et al. 1994; Longcope et al. 2001). A magnetic flux of $1.4 \times 10^{20} \mathrm{Mx}$ would be required under these conditions, an order of magnitude larger than the positive polarity flux. The uncertainty in the different values can not explain the disagreement, therefore we conclude that the heating of this BP can not be explained by a helicity injection process.

\section{Conclusions}

We have studied CDS wide slit movies of four coronal bright points (BPs) in three spectral lines: He I $584.34 \AA$, O v $629.73 \AA$ and Mg vII/Ix $368 \AA$. The BPs can be clearly identified in the $\mathrm{Mg}$ VII/IX images where they have a peak emission between 1.8-2.7 the flux of the surrounding corona. This identification is not straightforward for the cooler lines, where the emission and appearance can be similar to that of other network elements. The BPs show flux fluctuations with an amplitude generally ranging 4-20\% in He I $584.34 \AA, 10-20 \%$ in O v $629.73 \AA$ and $2-10 \%$ in Mg vII/IX $368 \AA$, although there are brighter events where the percentage could reach the $50 \%$ increase.

The wavelet analysis of the time-series confirms the oscillating nature of the BP's emission in EUV lines (Ugarte-Urra et al. 2004). We find wavetrains with periods ranging between $600-1100 \mathrm{~s}$ in all three lines. We find as well, in one of the BPs, periods as short as $236 \mathrm{~s}$. We notice that these periods lie in the period range of the chromospheric oscillations and we conclude that a fraction of the network bright points that are observed in the chromospheric network are most likely the cool footpoints of the loops that coronal BPs are made of.

In the case of one BP, for which MDI high resolution magnetograms were available, we have found that the increase in the EUV flux and its variability takes place as the magnetic polarities approach and the dominant (positive) flux decreases. Sudden magnetic flux decreases are observed at the same time as the oscillations in the EUV emission become significant. This would support a scenario where the waves, interpreted here in terms of global acoustic modes, are driven by magnetic reconnection events. However, Sweet-Parker and Petschek models do not seem to explain the typical small Doppler shift values characteristic of BPs. We believe that a new model is needed in order to explain these Doppler shift values in the context of magnetic reconnection, where waves play also a very important role.

Acknowledgements. Research at Armagh Observatory is grant-aided by the N. Ireland Dept. of Culture, Arts and Leisure. This work was supported by PPARC grant PPA/V/S/1999/00668. CDS, EIT and MDI are instruments onboard SoHO which is a project of international cooperation between ESA and NASA. We would like to thank the referee for useful comments and suggestions that helped us improve the presentation of the paper.

\section{References}

Andretta, V., Del Zanna, G., \& Jordan, S. D. 2003, A\&A, 400, 737 Aschwanden, M. J. 2003, in NATO Advanced Research Workshops: Turbulence, Waves and Instabilities in the Solar Plasma, 215

Banerjee, D., O'Shea, E., Doyle, J. G., \& Goossens, M. 2001, A\&A, 371,1137

Banerjee, D., O'Shea, E., Goossens, M., Doyle, J. G., \& Poedts, S. 2002, A\&A, 395, 263

Carlsson, M., Judge, P. G., \& Wilhelm, K. 1997, ApJ, 486, L63

Cauzzi, G., Falchi, A., \& Falciani, R. 2000, A\&A, 357, 1093

Chae, J., Wang, H., Qiu, J., et al. 2001, ApJ, 560, 476

Chae, J., Choi, B., \& Park, M. 2002a, J. Korean Astron. Soc., 35, 59 
Chae, J., Moon, Y., Wang, H., \& Yun, H. S. 2002b, Sol. Phys., 207, 73

Cook, J. W., Brueckner, G. E., \& Bartoe, J.-D. F. 1983, ApJ, 270, L89

Cooper, F. C., Nakariakov, V. M., \& Williams, D. R. 2003, A\&A, 409, 325

Curdt, W., \& Heinzel, P. 1998, ApJ, 503, L95

Dame, L., Gouttebroze, P., \& Malherbe, J.-M. 1984, A\&A, 130, 331

Deforest, C. E., \& Gurman, J. B. 1998, ApJ, 501, L217

De Moortel, I., \& Hood, A. W. 2000, A\&A, 363, 269

Deubner, F.-L., \& Fleck, B. 1990, A\&A, 228, 506

Doyle, J. G., van den Oord, G. H. J., O’Shea, E., \& Banerjee, D. 1999, A\&A, 347, 335

Egamberdiev, S. A. 1983, Soviet Astron. Lett., 9, 385

Gallagher, P. T., Phillips, K. J. H., Harra-Murnion, L. K., \& Keenan, F. P. 1998, A\&A, 335, 733

Gouttebroze, P., Vial, J.-C., Bocchialini, K., Lemaire, P., \& Leibacher, J. W. 1999, Sol. Phys., 184, 253

Habbal, S. R., \& Withbroe, G. L. 1981, Sol. Phys., 69, 77

Habbal, S. R., Withbroe, G. L., \& Dowdy, J. F. 1990, ApJ, 352, 333

Hagenaar, H. J. 2001, ApJ, 555, 448

Harrison, R. A., Sawyer, E. C., Carter, M. K., et al. 1995, Sol. Phys., 162,233

Hasan, S. S., \& Kalkofen, W. 1999, ApJ, 519, 899

Kalkofen, W. 1997, ApJ, 486, L145

Krijger, J. M., Rutten, R. J., Lites, B. W., et al. 2001, A\&A, 379, 1052

Lites, B. W., Rutten, R. J., \& Kalkofen, W. 1993, ApJ, 414, 345

Longcope, D. W. 1998, ApJ, 507, 433

Longcope, D. W., Kankelborg, C. C., Nelson, J. L., \& Pevtsov, A. A. 2001, ApJ, 553, 429

Lou, Y. 1995, MNRAS, 274, L1

Madjarska, M. S., \& Doyle, J. G. 2002, A\&A, 382, 319

Madjarska, M. S., \& Doyle, J. G. 2003, A\&A, 403, 731

Madjarska, M. S., Doyle, J. G., Teriaca, L., \& Banerjee, D. 2003, A\&A, 398, 775
Marsh, M. S., Walsh, R. W., \& Bromage, B. J. I. 2002, A\&A, 393, 649

Mazzotta, P., Mazzitelli, G., Colafrancesco, S., \& Vittorio, N. 1998, A\&AS, 133, 403

McAteer, R. T. J., Gallagher, P. T., Williams, D. R., et al. 2002, ApJ, 567, L165

McAteer, R. T. J., Gallagher, P. T., Williams, D. R., et al. 2003, ApJ, 587,806

McAteer, R. T. J., Gallagher, P. T., Bloomfield, D. S., et al. 2004, ApJ, 602, 436

Musielak, Z. E., \& Ulmschneider, P. 2003, A\&A, 406, 725

Nakariakov, V. M., Verwichte, E., Berghmans, D., \& Robbrecht, E. 2000, A\&A, 362, 1151

Nakariakov, V. M., Tsiklauri, D., Kelly, A., Arber, T. D., \& Aschwanden, M. J. 2004, A\&A, 414, L25

Nindos, A., \& Zirin, H. 1998, Sol. Phys., 179, 253

Ofman, L., \& Wang, T. 2002, ApJ, 580, L85

O'Shea, E., Banerjee, D., Doyle, J. G., Fleck, B., \& Murtagh, F. 2001, A\&A, 368, 1095

Parnell, C. E. 2002, MNRAS, 335, 389

Pauluhn, A., Rüedi, I., Solanki, S. K., et al. 1999, Appl. Opt., 38, 7035

Popescu, M. D., Doyle, J. G., \& Xia, L. 2004, A\&A, in press

Priest, E. R., Parnell, C. E., \& Martin, S. F. 1994, ApJ, 427, 459

Priest, E. R., Longcope, D. W., \& Titov, V. S. 2003, ApJ, 598, 667

Sheeley, N. R., \& Golub, L. 1979, Sol. Phys., 63, 119

Teriaca, L., Banerjee, D., \& Doyle, J. G. 1999, A\&A, 349, 636

Thompson, W. T. 2000, CDS Software Note $\mathrm{N}^{\circ} 49$

Torrence, C., \& Compo, G. P. 1998, Bull. Amer. Meteor. Soc., 79, 61 Ugarte-Urra, I., Doyle, J. G., Madjarska, M. S., \& O’Shea, E. 2004, A\&A, 418, 313

Wilhelm, K., \& Kalkofen, W. 2003, A\&A, 408, 1137

Xia, L. D., Marsch, E., \& Curdt, W. 2003, A\&A, 399, L5

Young, P. R., Del Zanna, G., Landi, E., et al. 2003, ApJS, 144, 135

Zhang, J., Kundu, M. R., \& White, S. M. 2001, Sol. Phys., 198, 347 